TIPA. Travaux interdisciplinaires sur la parole et le langage

34 | 2018

La langue des signes, c'est comme ça

\title{
Une approche typologique de la langue des signes française
}

A typological approach of French Sign Language

Nicolas Tournadre et Mélanie Hamm

\section{OpenEdition}

1 Journals

Édition électronique

URL : http://journals.openedition.org/tipa/2568

DOI : $10.4000 /$ tipa.2568

ISSN : 2264-7082

Éditeur

Laboratoire Parole et Langage

Référence électronique

Nicolas Tournadre et Mélanie Hamm, «Une approche typologique de la langue des signes française », TIPA. Travaux interdisciplinaires sur la parole et le langage [En ligne], 34 | 2018, mis en ligne le 08 février 2019, consulté le 10 octobre 2020. URL : http://journals.openedition.org/tipa/2568 ; DOI : https:// doi.org/10.4000/tipa.2568

Ce document a été généré automatiquement le 10 octobre 2020

La revue TIPA. Travaux interdisciplinaires sur la parole et le langage est mise à disposition selon les termes de la licence Creative Commons Attribution - Pas d'Utilisation Commerciale - Pas de Modification 4.0 International. 


\title{
Une approche typologique de la langue des signes française
}

\author{
A typological approach of French Sign Language
}

Nicolas Tournadre et Mélanie Hamm

Ce média ne peut être affiché ici. Veuillez vous reporter à l'édition en ligne http:// journals.openedition.org/tipa/2568

\section{Introduction}

2 Les langues gestuelles ou langues signées (LS) n'ont été reconnues comme des langues à part entière que tardivement. L'étude linguistique fondatrice est attribuée à Stokoe (1960) qui démontra le caractère doublement articulé de l'American Sign Language (ASL), jusque-là considéré comme du mime ou de la pantomime (Millet, 2004). La recherche dans ce domaine et la reconnaissance des langues des signes (LS) ont considérablement progressé depuis. Toutefois, on ne compte actuellement en Europe que quatre pays reconnaissant une langue des signes dans leur Constitution : l'Autriche depuis 2005, la Finlande depuis 1995, la Hongrie depuis 2011 et le Portugal depuis $1997^{1}$. Certains linguistes considèrent que les langues des signes ne sont pas vraiment des langues naturelles ni même des langues à part entière. Citons par exemple le propos de Kirtchuk (2016: 9) qui préfère utiliser le mot «langue» entre guillemets (sign «language»):

It [sign « language »] is meant to compensate plain language ; it is in a way close to the flag language that was used for naval communication when distances were too great to communicate otherwise ${ }^{2}$.

Voici un autre exemple :

Des codes secondaires comme le morse, le sémaphore, le braille, le langage des signes, etc., sont calqués sur le langage parlé ou la langue écrite mais seul le langage parlé est universellement attesté, écrit Schön (2008:6). 
Les langues des signes sont pourtant bel et bien des langues au sens où elles ont la même efficacité communicative que les langues vocales, et sont aussi comme ces dernières des langues naturelles ${ }^{3}$. Si elles diffèrent des langues vocales par leur modalité visuo-gestuelle, elles n'en possèdent pas moins un lexique, une grammaire et une " chérologie » qui correspond à la phonologie des langues vocales ${ }^{4}$. Tout comme les créoles fournissent de précieuses informations sur la formation et les caractéristiques des langues humaines du fait de leur genèse spécifique, les langues des signes par leur modalité singulière interpellent certains linguistes et notamment ceux qui s'intéressent à la diversité des langues.

5 L'objectif ici est de montrer qu'au-delà de ses spécificités structurelles et fonctionnelles, la langue des signes partage des caractéristiques typologiques que l'on retrouve dans certaines familles de langues vocales ainsi que dans des langues écrites de type logographiques. Nous prendrons l'exemple de la langue des signes française $(\mathrm{LSF})^{5}$. Dans un premier temps, nous tenterons de décrire certaines spécificités sociolinguistiques et structurelles de la langue des signes. Dans un deuxième temps, nous présenterons les classes lexicales (nom, verbe, adjectif, etc.) et les caractéristiques typologiques majeures de la LSF.

\section{Approches génétique et typologique}

6 On dénombrerait actuellement 136 langues des signes à travers le monde ${ }^{6}$. Wittmann (1991) propose la classification des langues des signes dans les familles suivantes: allemande, britannique, française, lyonnaise et japonaise, sans compter les très nombreux isolats. D'un point de vue génétique, la cladistique des langues des signes n'est pas encore suffisamment développée. Pour ce qui concerne les langues orales, les classifications génétiques reposent notamment sur la notion d'intercompréhension et sur l'existence de proto-langue(s) attestées ou reconstruites. Outre les critères lexicaux, la grammaire joue un rôle fondamental dans ces classifications, et elle est perçue comme beaucoup plus significative que le lexique. En effet, ce dernier peut être largement emprunté à une langue d'une autre famille génétiquement apparentée ou non : ainsi, l'anglais qui a emprunté environ la moitié (voire les deux tiers de son vocabulaire selon Walter \& Lorca, 2001) au franco-normand et au latin est, malgré cet apport, unanimement classé comme une langue germanique. De même, le persan, langue indo-européenne (de la branche indo-iranienne), qui a emprunté également environ la moitié de son vocabulaire à l'arabe (langue sémitique) apparaît systématiquement classé comme une langue indo-européenne. Dans le cas de l'anglais comme dans celui du persan, les classifications génétiques considèrent la grammaire comme un critère fondamental, et en tout cas plus déterminant que celui du lexique. La classification des langues des signes en famille(s) linguistique(s) devrait elle aussi se fonder sur des critères grammaticaux et nécessite un travail de reconstruction analogue à celui qui a été réalisé notamment pour les familles indo-européenne, sémitique et bantoue.

7 Toutefois, deux observations peuvent être faites quant aux langues des signes actuelles. Tout d'abord, il existe un certain nombre de langues des signes qui n'admettent pas d'intercompréhension. Par exemple, les langues des signes française, britannique, chinoise et tibétaine, etc. ne permettent qu'une communication très limitée au niveau de la conversation courante, sans parler d'un niveau avancé ${ }^{7}$. Pour expliquer ce 
phénomène, il suffit de rappeler que les systèmes de signes sont largement fondés sur des symboles culturels ayant souvent une valeur historique ${ }^{8}$. De plus, les langues des signes présentent aussi des différences substantielles dans leur grammaire ${ }^{9}$ (voir Zeshan, 2006). Néanmoins, il est nécessaire de rappeler - et de souligner - qu'une communication de base entre deux langues des signes même très éloignées est beaucoup plus rapide qu'entre des langues vocales distinctes ${ }^{10}$.

Concernant la typologie des LS, on constate l'apparition d'un certain nombre de travaux à ce sujet depuis le début des années 2000. Toutefois, les recherches à grande échelle dans les domaines génétiques et typologiques en sont encore à leurs débuts comme le rappelle Zeshan (2006) :

Sign language linguistics so far has not developed any principled methods of establishing family membership for a particular sign language ${ }^{11}$, reconstructing earlier forms of sign languages, or setting up family trees for sign languages. Therefore, any effort towards a genetically balanced sample of sign languages is again defeated from the very beginning, and all that can be done is to draw attention to this current limitation in sign language typology research in general $[\ldots]^{12}$.

\section{Rattachement géographique et relations à la langue écrite}

9 Certains phénomènes sociolinguistiques semblent à première vue spécifiques aux langues signées. C'est le cas par exemple de l'absence de rattachement à un territoire géographique particulier (voir notamment Cuxac \& Antinoro Pizzuto, 2010: 41; Mugnier, Estève \& Millet, 2016). Toutefois, on peut identifier des pôles communautaires et institutionnels qui sont à l'origine de variations dialectales dans les LS. Donc là encore, il faut relativiser la singularité sociolinguistique des langues des signes.

Parmi les autres phénomènes sociolinguistiques, on peut mentionner l'existence d'une forme de diglossie mettant en relation le «français signé " et la langue des signes française (voir notamment Mottez, 1976, 1977 ; Cuxac, 1993 ; Voisin, 2008: 80). Cette dénomination de "français signé » désigne en réalité non pas une forme de français visuo-gestuel mais une langue des signes francisée, c'est-à-dire une langue des signes influencée principalement par la syntaxe voire la morphologie du français et que l'on pourrait nommer langue des signes à syntaxe française (LSSF) ${ }^{13}$. La LSF et la LSSF forment en réalité un continuum inter-linguistique ${ }^{14}$. En outre, la langue des signes française fait de nombreux emprunts au français écrit (voire à d'autres langues écrites, comme à l'anglais écrit), grâce à son alphabet dactylologique notamment (voir entre autres Garcia \& Perini, 2010). L'alphabet dactylologique n'est pas seulement utilisé pour citer la langue française sous une forme écrite; des configurations dactylologiques sont aussi empruntées pour former certains signes standard qui utilisent la première lettre d'un mot pour représenter le signe. Ces signes sont appelés «signes initialisés»; par exemple : deux C pour COUSIN, deux L pour LANGUE, un o pour ONCLE, deuX R pour RUE, etc. (voir notamment Garcia \& Perini, 2010).

11 La plupart des signes sont issus de la grande iconicité (voir section 4.3.). Y a-t-il un système correspondant à la grande iconicité dans les langues vocales? La réponse est clairement négative. On peut toutefois faire une analogie entre la grande iconicité et le discours direct. En effet, ce type de discours intègre des onomatopées, des idéophones 
et imite la prosodie et la voix du discours rapporté. L'existence de divers modes de productions du sens dans les langues vocales n'est pas totalement absent des langues vocales, si l'on adopte le point de vue des théories multimodales et l'on intègre la gestualité co-verbale (Boutet \& Cuxac, 2008). Cette comparaison entre la grande iconicité et le discours direct est toutefois très imparfaite.

Il est très complexe de noter par écrit une langue des signes. Aucune langue des signes n'est transcrite par écrit ${ }^{15}$. Que peut-on dire à propos du rapport des sourds à l'écrit? Une proportion élevée d'adultes sourds sont en difficulté avec l'écrit ${ }^{16}$. Pourtant, pour les sourds, y compris ceux qui sont peu lettrés voire illettrés, l'écriture joue un rôle d'autant plus important dans l'éducation et la formation que les informations transmises par l'oralité sont restreintes (Hamm, 2008, 2010, 2012). Si l'on omet le cas des écritures idéographiques, comme le japonais et le chinois, la quasi-totalité des écritures dans le monde reflète à des degrés divers les sons correspondant à la prononciation des $\operatorname{mots}^{17}$. Or dans le cas des communautés de personnes sourdes possédant à la fois la langue des signes française et le français écrit, celles-ci n'entretiennent presque aucun lien direct. Les langues des signes sont des langues visuo-gestuelles et donc silencieuses, elles ne reposent ni sur la phonétique, ni sur la phonologie de la langue française. Les signes dactylographiés et les signes initialisés jouent un rôle marginal dans le fonctionnement du système de la langue des signes, même s'ils peuvent être fréquents dans certains types de discours.

Hormis quelques discours signés particuliers ${ }^{18}$, la langue des signes française est plus proche du chinois écrit que du français écrit. Par exemple, MONTAGNE en langue des signes : deux mains plates qui montent et se rejoignent, formant ainsi un triangle ; 山en chinois écrit: signe qui stylise trois sommets d'une montagne. Le chinois écrit contemporain (simplifié ou traditionnel) n'est certes plus un système pictographique, mais il en garde de nombreux traces (Yau, 1992, 2004 ; Tournadre, 2014b/2016). À l'inverse, les langues des signes restent largement motivées d'un point de vue iconique. Toutefois, la motivation d'un certain nombre de signes standard est devenue opaque. Les travaux sur l'American Sign Language (ASL) de Klima et Bellugi (1979: 22-24), par exemple, montrent que des non-signeurs ne devinent presque jamais la signification d'un signe gestuel alors qu'ils devinent presque toujours celle d'une pantomime ${ }^{19}$.

\section{Les caractéristiques fondamentales du signe}

14 Nous rappellerons ici les caractéristiques fondamentales du signe, la particularité de l'espace de signation et la distinction entre signes standard (ou signes conventionnels) et "transferts» selon Cuxac (2000), l'auteur de ce concept (voir section 4.3.). Cette approche est appelée « modèle sémiologique » (voir Cuxac \& Antinoro Pizzuto, 2010).

\subsection{Les paramètres}

Les signes sont des unités significatives qui se composent de quatre paramètres manuels et de quatre paramètres non manuels. Les premiers sont : la configuration de la main, l'orientation de la main, l'emplacement et le mouvement. Les seconds types de paramètres sont le regard, l'expression faciale, la posture corporelle - absolument cruciaux - et le mouvement labial (Cuxac, 2000) ${ }^{20}$. 

la phonologie et à la morphologie des langues vocales: phonème et morphème. Toutefois, il existe des alternatives : les correspondants des phonèmes sont parfois appelés " chérèmes 》 (selon la terminologie de Stokoe, 1960) ou «gestèmes » (Cuxac, 2000 ; Voisin, 2008), mais le terme d'« unités paramétriques » est aussi utilisée (Cuxac, 2000 ; Millet, 2004; Voisin, 2008). Les unités morphologiques correspondant aux morphèmes ont été appelées « kinèmes » par Stokoe (1960), mais ce terme n'est plus guère utilisée ${ }^{21}$. Les tenants du "modèle sémiologique» remettent en question l'idée qu'il y a une organisation de type phonologique dans les langues des signes ${ }^{22}$.

La question de la pertinence de la double articulation pour les langues des signes et de l'équivalence des unités paramétriques aux phonèmes fait encore l'objet de débats (Cuxac, 2000), même si la plupart des chercheurs défendent l'idée d'une double articulation. L'adaptation des concepts dérivés de la phonologie aux LS nécessite une révision si complète des concepts linguistiques que l'on peut se demander si l'analogie conserve toute sa pertinence. Les notions de phonème et de syllabe sont fondamentales dans les langues vocales et sont profondément imprégnées de leur nature sonore ; ainsi, même lorsqu'on les utilise à un niveau très abstrait, elles sont susceptibles d'introduire des distorsions dans la description d'un système foncièrement visuel quadridimensionnel et dynamique. Contrairement à la " phonologie ", l'existence d'une morphologie en LS n'est pas en soi problématique, puisqu'il est également possible d'analyser la forme des « mots ». Celle-ci correspond à une forme visuelle dans le cas des langues des signes, tandis que les langues vocales possèdent des formes sonores. La morphologie s'intéresse tout particulièrement à la variation des formes ainsi qu'à leur composition.

\subsection{L'espace de signation}

Les signes sont réalisés dans un espace de signation situé devant le signeur. Une symbolique spatiale est en rapport avec les différentes parties du visage et du buste; par exemple, IDÉE, IMAGINER, PENSER : signes qui se réalisent au-dessus de la tête ; AIMER CONTENT, DÉSOLÉ: signes qui se font au niveau de la poitrine. C'est notamment cette symbolique spatiale qui permet une communication de base entre sourds issus de divers pays malgré les différences linguistiques et culturelles (revoir section 2.). L'emplacement de la réalisation du signe a une incidence sur l'interprétation. Cuxac (1998) fournit l'exemple oUVRIR FENÊTRE qui, déplacé au niveau du crâne, signifie « ouvrir (la fenêtre) de son esprit ». Autre exemple : HANDICAPÉ signé au niveau de la tête du signeur veut dire «handicapé de la tête ». Par conséquent, on peut relever différents jeux de signes comme ÉTOILE, PAIX, SOLEIL ou SOURIRE qui se réalisent au niveau du cœur et sont ainsi très significatifs.

19 A l'instar de ce qui se passe dans les langues vocales, la distance entre les signeurs est un élément culturel important: une certaine distance indique le respect; un rapprochement des signeurs signale une communication intime, mais agressive en cas de rapprochement très intimidant ${ }^{23}$.

TIPA. Travaux interdisciplinaires sur la parole et le langage, 34 | 2018 


\subsection{Les signes standard et les transferts}

20

caractéristique majeure des langues signées est production du sens structurellement très différents. Ces deux modes ont été caractérisés par Cuxac (2000) comme «deux visées » : une visée illustrative qui utilise « les structures de grande iconicité ou les transferts » et une structure non illustrative qui utilise les « signes standard, les pointages et la dactylologie ».

21 Les signes standard et les transferts sont tous les deux motivés par l'iconicité mais dans le cas des signes standard, les motivations à l'origine de la forme du signe relèvent de l'étymologie et ne sont pas forcément évidentes. Le discours de grande iconicité consiste à dire en montrant : c'est grand « comme ça » ou «ça s'est passé comme ça » (Cuxac, $2000: 23)^{24}$. La grande iconicité est constituée de plusieurs types de transfert : a) des transferts de taille ou de forme (TTF) qui n'impliquent ni procès ni actant ; $b$ ) des transferts de situation (TS) qui représentent une "scène comme vue de loin » et implique actant(s) et procès; c) des transferts de personne (TP) dans lesquels le locuteur devient l'entité qu'il décrit ; d) les doubles transferts, qui peuvent impliquer à la fois un transfert de personne et de situation (Cuxac, 1996, 2000 ; Sallandre, 2001, 2003). lexèmes des langues vocales. Quant aux unités de transfert (UT), celles-ci n'ont pas vraiment d'équivalents en langue vocale, comme le rappelle Garcia (2016 : 247) :

Les UT, dont le sens est construit, dans la simultanéité, par l'ensemble des paramètres manuels et corporels, sont plutôt en termes de contenu conceptuel l'équivalent d'une proposition ou d'une phrase en LV. Un TS par exemple pourrait équivaloir à quelque chose comme ceci : «forme verticale mince et allongée se déplaçant lentement vers une forme ovoïde horizontale et fixe ».

À côté des pointages et du regard, les unités de transfert, notamment les TTF (voir section 8.5.), jouent un rôle important dans la grammaire de la LSF et plus généralement des langues signées. Il est important de préciser que les deux modes de production du sens sont tout à fait imbriqués dans la conversation courante. En effet, dans un discours naturel entre deux sourds natifs de langue des signes française, on constate un va-et-vient constant entre les deux visées. La frontière entre les deux visées dans la réalisation de certains signes peut être relativement ténue.

Comme écrit précédemment, Cuxac (2000) réunit les signes standard, le pointage et la dactylologie sous la visée non illustrative. Il nous semble utile de distinguer nettement les signes de l'alphabet dactylologique des signes standard. En effet, l'alphabet dactylologique permet au signeur de «transcrire » du français écrit (revoir section 3.) ; il constitue par conséquent un système radicalement différent de celui correspondant aux signes standard. Si l'on adopte cette position, on considérera que la LSF possède en réalité trois systèmes reposant sur des fonctionnements différents : les structures de grande iconicité, les signes standard et l'alphabet dactylologique. Ces trois systèmes interagissent dans le discours signé.

Les langues des signes ne sont pas les seules à utiliser des systèmes différents à l'intérieur d'une même langue. Certaines langues écrites ont recours à plusieurs systèmes; l'écriture japonaise, par exemple, est constituée de quatre systèmes différents: les kanji ou caractères sino-japonais de type «idéographique » ou idéophonographique, les deux syllabaires hiragana et katakana et enfin, marginalement, l'alphabet latin ${ }^{25}$.

TIPA. Travaux interdisciplinaires sur la parole et le langage, 34 | 2018 

et n'évoquerons que marginalement les structures de transfert, laissant de côté l'alphabet dactylologique. Nous sommes conscients que cela est réducteur dans la mesure où les unités de transfert peuvent représenter entre 30 à $70 \%$ des unités d'un discours (Sallandre, 2003 ; Meurant, 2008; Garcia, 2010, 2016). Ce choix est motivé par des raisons typologiques et la volonté de comparer les langues signées avec les langues vocales. Or, comme les langues vocales ne disposent pas d'UT, ces dernières ne peuvent servir de base à une comparaison.

Concernant le lexique standard en LSF, rapportons ici une remarque de Cuxac et Pizzuto (2010) :

Comparés aux dictionnaires des LV à leurs dizaines de milliers de mots, les dictionnaires des LS, même les plus élaborés, ne dépassent généralement pas 4 à 5000 signes. Or l'interprétation en LSF par exemple montre que toutes sortes de contenus complexes sont traduits sans déperdition de sens.

Le nombre relativement limité de signes peut engendrer l'idée fausse d'une certaine pauvreté sémantique. Ce serait considérer que la sémantique d'une langue est essentiellement mesurée par le nombre de lexèmes et oublier que d'autres facteurs jouent un rôle essentiel dans la construction du sens. Or le caractère limité du lexique est aussi attesté dans des langues vocales, pourtant susceptibles de traduire des contenus complexes. C'est le cas par exemple des langues inuites (Tersis, 2008). D'après Lavie (2007), le nombre de bases lexicales est réduit, (env. 2000) ce qui est compensé par la grande productivité morphologique. Ces langues, en effet, ont recours à des stratégies polysynthétiques qui démultiplient les sens possibles. Quant aux LS, celles-ci utilisent, elles aussi, diverses stratégies morphologiques permettant de multiplier les unités lexicales à partir d'un nombre limité d'unités de base. Mentionnons par exemple l'utilisation de la composition nominale en LSF : MÉDECIN-PEAU pour « dermatologue » ou PIANO-VIOLON-FLÛTE-ETC. pour " orchestre ». La composition nominale est évidemment l'une des stratégies dominantes dans les langues du monde ${ }^{26}$.

\section{Les ordres syntaxiques privilégiés en LSF}

La question de l'ordre des signes dans les LS a fait l'objet de débats, mais il est maintenant établi qu'il existe des ordres privilégiés. Par exemple, en LSF, le verbe a tendance à apparaître en position finale. C'est l'ordre Agent-Patient-Prédicat («SOV ») qui semble le plus spontané dans cette langue mais ce n'est pas le seul attesté (Yau, 1993 ; Cuxac, 2000; Lejeune, 2004; Voisin, 2008; etc.). Parmi les 7000 langues du monde, cet ordre syntaxique est l'ordre le plus fréquent à côté de l'ordre AgentPrédicat-Patient ( $(\mathrm{SVO} »)^{27}$. Le fait que plusieurs ordres syntaxiques de base soient attestés n'est en rien une spécificité de la LSF. Certaines langues vocales, particulièrement les langues riches en morphologie comme le basque, le russe ou le latin ont une grande flexibilité dans l'ordre des mots qui est alors utilisée à des fins pragmatiques (notamment pour l'opposition thème-rhème).

À côté de l'ordre Agent-Patient-Prédicat ("SOV »), Cuxac (2000: 208) mentionne les ordres suivants : Patient-Agent-Prédicat ( OSV ») et Agent-Prédicat-Patient ( SVO ») $)^{28}$. L'ordre Agent-Prédicat-Patient reflète plus l'ordre du français signé (Voisin, $2008: 77$; voir aussi Yau, 1992: 87-89). Quoi qu'il en soit, la réalisation de ces ordres dépend de la spatialisation des actants (c'est-à-dire de l'existence de pointages) ainsi que de

TIPA. Travaux interdisciplinaires sur la parole et le langage, 34 | 2018 
paramètres sémantiques, notamment du caractère animé ou non des actants ${ }^{29}$. Cuxac (2000: ibid.) précise que pour un locuteur droitier, la relation s'établit en spatialisant l'agent à droite, le patient à gauche. En langue des signes, les relations sémantico-syntaxiques sont par conséquent exprimées par la spatialisation des entités du discours (voir notamment Yau, $1992: 121)$.

Les langues en général diffèrent dans la pondération qu'elles accordent à la sémantique et à la syntaxe. Il est clair que la sémantique joue un rôle essentiel et doit être privilégiée dans les analyses de la langue des signes française, comme l'a montré Cuxac (2000). Cela ne signifie pas que la syntaxe joue un rôle négligeable en LSF, mais cela signifie qu'il faut se garder d'appliquer des fonctions syntaxiques (Sujet, Objet, etc.) dans des langues pour lesquelles, les rôles grammaticaux de type sémantique (comme l'Agent, Patient) ou pragmatique (Topique, Focus, etc.) permettent de mieux rendre compte des faits. Ainsi, en tibétain comme en chinois, la fonction de sujet ne peut s'appliquer facilement et est abandonnée par de nombreux chercheurs (LaPolla, 1993 ; Tournadre, 2014a). Il en va de même pour la notion de pivot syntaxique. Dans certaines langues, le sujet fonctionne comme un pivot syntaxique et la syntaxe prévaut sur les interprétations sémantiques les plus évidentes. Dans l'énoncé français «le père a frappé l'enfant et il a pleuré » est ambigu : la personne qui pleure peut être "l'enfant » mais aussi le "père ». Toutefois, cette ambiguïté est levée par la syntaxe du français lorsque l'on omet le pronom anaphorique "le père a frappé l'enfant et a pleuré », le français qui associe le pivot syntaxique au sujet impose la lecture selon laquelle c'est le père et non l'enfant qui a pleuré (même si cette interprétation est peu probable). Examinons les équivalents de ces énoncés en LSF $^{30}$ :

(1) PÈRE (localisation à droite) + ENFANT (localisation à gauche) + FRAPPER (l'action frapper va de père à enfant) // LUI (pointage sur l'enfant) + PLEURER (« le père a frappé l'enfant ; l'enfant a pleuré ») $)^{31}$

Contrairement au français, cette phrase signée ne comporte pas d'ambiguïté, car le pointage sur le locus de l'enfant indique que c'est ce dernier qui a pleuré. Toutefois, il est possible, comme en français, d'omettre le « pronom de rappel » correspondant au pointage :

(2) PÈRE (localisation à droite) + ENFANT (localisation à gauche) + FRAPPER (l'action frapper va de père à enfant) // PLEURER (« le père a frappé l'enfant ; l'enfant a pleuré»)

Cet énoncé est interprété ainsi : «le père a frappé l'enfant ${ }_{(\mathrm{i})}$ et $\mathrm{il}_{(\mathrm{i})}$ a pleuré ». Il n'y a donc pas de pivot syntaxique, comme en français ou dans d'autres langues européennes. Cependant, ce procédé est plus complexe, dans la mesure où lorsque le pointage est omis, le signeur peut, en effectuant le signe PLEURER, pencher légèrement la tête en direction du locus de l'enfant, mouvement qui est parfois imperceptible, surtout pour les signeurs débutants. La LSF, qui connaît une réelle souplesse syntaxique, permet d'autres variantes de ce même énoncé. On peut en effet énoncer le même propos de la façon suivante : 
(3) PÈRE (localisation à droite) + FRAPPER // ENFANT (localisation à gauche) + PLEURER (

« le père a frappé ; l'enfant a pleuré »)

En outre, la motivation sémantico-cognitive en LSF explique la préférence de certains ordres syntaxiques, notamment les ordres localisant - localisé, objet repère - objet repéré, stable - mobile, contenant - contenu et de façon plus générale, l'ordre fond-figure comme l'a montré Cuxac (2000: 183). Les langues signées sont des «langues situationnelles », dans la mesure où la situation d'énonciation fournit souvent de nombreuses informations qui sont intégrées dans la communication.

\section{Les procédés morphologiques majeurs}

Les procédés morphologiques utilisés en LSF ont-ils des points communs avec ceux des langues vocales? Parmi les procédés fréquemment attestés dans les langues vocales, on trouve : l'affixation, la cliticisation, la réduplication, la conversion, l'alternance, la métathèse, la soustraction, ou encore l'accent tonique et le ton. Concernant les procédés impliquant deux lexèmes, on peut également mentionner la composition, la supplétion, le téléscopage, l'amalgame ou encore l'acronymie. Certains tels que les procédés suprasegmentaux (l'accent tonique ou le ton) ne sont pas universels.

Qu'en est-il en langue des signes française? Certains procédés, fréquents dans les langues orales, sont bien attestés en LSF. C'est le cas par exemple de la réduplication qui joue un rôle important au niveau grammatical pour indiquer le pluriel des noms ou encore l'aspect verbal. On peut en outre mentionner la conversion et la supplétion ${ }^{32}$. L'emploi assez important de la supplétion (notamment avec les formes négatives) n'est pas sans rappeler l'utilisation de la supplétion en russe (pour les verbes de mouvement et le pluriel de certains noms) ou encore les langues tibétiques (pour les temps et modes verbaux). Nous laissons ici en suspens les cas de l'affixation et de la cliticisation ${ }^{33}$.

Pour des raisons dues à son mode opératoire et pour des raisons d'ordre iconique, les langues des signes ont développé des procédés morphologiques spécifiques. Ainsi, la direction du signe et l'inversion du signe qui sont essentielles en LSF semblent ne pas avoir d'analogie dans les langues vocales. Illustrons tout d'abord le cas de la direction du signe. D'un point de vue morphologique, les deux énoncés suivants ne diffèrent que par l'orientation du signe qui pointe dans deux directions différentes :

(4) DEMANDER (le signe « demander » - représenté par deux mains jointes avec au

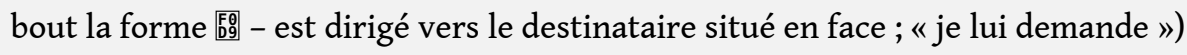

(4') DEMANDER (le signe « demander » - représenté par deux mains jointes avec au bout la forme ' - est dirigé vers une troisième personne présente ou imaginée, localisée à droite ou à gauche du signeur ; « je lui demande »)

L'inversion est un cas particulier dans lequel la direction est inversée, formant ainsi un nouveau signe. Par exemple : 
(5) DEMANDER (le signe « demander » est inversé par rapport au signe présenté dans l'exemple (4) - il représente avec les deux mains jointes la forme ${ }^{\vee}$ que le signeur pointe vers lui-même ; « il me demande ») que les recherches aient fait l'économie de cette question, écrit Risler (2007). L'état des recherches concernant la question de l'existence des classes lexicales - nom, verbe, adjectif, adverbe, adposition - en LSF, particulièrement en ce qui concerne le lexique standard, est encore à ses débuts. Dans la plupart des langues, l'opposition verbonominale ne pose pas de problème fondamental, même si dans certains cas, comme les langues inuites (Tersis, 2008) ou le mwotlap (François, 2003), la distinction n'est pas toujours facile à établir. nom et le verbe se manifeste généralement par la nature du mouvement effectué pour former le signe (Risler, 2002) ${ }^{35}$. Ce serait aussi le cas pour d'autres langues des signes. En ASL, le mouvement du signe désignant un nom est souvent doublé, alors que celui qui se réfère au verbe correspondant est un mouvement simple. En outre, comme dans les langues vocales, la possibilité d'associer une unité lexicale à des opérateurs de temps ou d'espace induit une interprétation verbale tandis qu'un contexte où apparaissent des marques de possessions conduit à une lecture nominale ${ }^{36}$.

\section{Quelques caractéristiques typologiques majeures de la LSF}

Quelles sont les principales caractéristiques typologiques de la LSF ? Peut-on les mettre en perspective avec celles qui sont attestées dans les langues vocales? 


\subsection{Absence de genre grammatical}

Il n'y a pas d'article défini ou indéfini en langue des signes française. Les langues vocales dépourvues d'articles sont aussi nombreuses (Dryer, 2013a). En LSF, le démonstratif et les adjectifs possessifs qui indiquent aussi la définitude sont normalement placés derrière le nom ${ }^{37}$, comme en tibétain ou en indonésien, et non devant le nom comme en français ou en chinois ; par exemple : ENFANT + LUI (pointage sur enfant ; « cet enfant ») ou PÈRE + MON (possessif de la première personne du singulier, poss. $1 \mathrm{sg}$; « mon père »).

\subsection{Optionalité du nombre}

47 Le nombre n'est pas toujours obligatoire en LSF, par exemple ENFANT + DORMIR peut indiquer qu' un ou plusieurs enfants dorment». C'est aussi le cas dans certaines langues vocales, comme le chinois, le tibétain ou le japonais.

\subsection{Catégories pronominales}

48 À l'instar des langues orales, la LSF distingue divers types de pronoms: personnels, démonstratifs, possessifs, interrogatifs. Les pointages et le regard permettent d'opposer les trois personnes : élocutif (première personne), allocutif (deuxième personne) et allocutif (troisième personne). La première personne est généralement absente et indiquée par défaut ${ }^{38}$. C'est le cas avec de nombreux signes comme : AVOIR, BOIRE, LIRE, MANGER, OUBLIER, SAVOIR, etc. Par contre, l'expressivité associée au visage est souvent plus marquée avec la première personne.

À ce premier paradigme des trois personnes, s'ajoute la possibilité d'utiliser des formes duelles (« nous deux », « vous deux », « eux deux ») ou trielles (« nous trois », " vous trois », " eux trois»). De plus, un signeur peut composer des pronoms personnels désignant des groupes supérieurs à trois et comprenant jusqu'à dix personnes (correspondant aux doigts des deux mains) ${ }^{39}$. En outre, grâce à l'orientation des signes, la LSF opère une distinction entre les formes de première personne du pluriel «nous » inclusif et d'exclusif ${ }^{40}$. Elle peut combiner cette opposition au marquage du duel, triel, etc. et est donc susceptible de distinguer entre duel inclusif vs duel exclusif, triel inclusif vs triel exclusif, etc.

50 Notons que d'un point de vue formel, les pronoms personnels ne sont pas différents des démonstratifs ( $\mathrm{IL}=$ CELUI-LÀ). Il est fréquent dans les langues du monde que les pronoms 
de la troisième personne soient identiques à une forme de démonstratifs. C'est le cas par exemple en coréen, en mongol, dans de nombreuses langues indiennes ou encore en amdo (Jacquesson, 2008).

51 Par contre, la LSF ne semble pas posséder de pronom relatif au sens strict. Elle utilise à la place des pointages, qui ont une valeur de démonstratifs. L'absence de pronom relatif et l'utilisation de démonstratifs pour cette fonction sont aussi attestées dans les langues vocales ${ }^{41}$.

52 Notons au passage qu'il n'existe pas de vouvoiement à proprement parler, mais les gestes réalisés avec élégance sont susceptibles de véhiculer une relation respectueuse et une certaine politesse. Dans ce cas, les gestes sont effectués plus lentement et de façon précise. Le respect est signalé par une distance entre les deux signeurs. Inversement, si une personne se met en colère ou adopte une attitude offensante, les gestes sont réalisés de façon plus esquissée et beaucoup plus rapide, la distance avec l'interlocuteur étant réduite (revoir section 4.2.). Les pointages en direction d'une personne présente sont considérés comme impolis; l'on utilise à cette fin le signe FLÈCHE qui symbolise une flèche et permet une ostension discrète.

\subsection{Présence de classificateurs ou proformes}

53 Un classificateur ou proforme est un signe iconique (revoir section 4.3.); il représente généralement la forme ou le mouvement d'une chose, d'un animal ou d'une personne en déplacement. Le classificateur fonctionne notamment comme un pronom de reprise ; il permet une économie dans le discours: il remplace un nom, objet, animal ou personne, signé auparavant, mais de plus il en montre la forme. C'est donc une sorte de « super-pronom » (Girod, 1998 : 127). Reprenons ici l'exemple fourni par Millet (2004) :

Supposons que l'on veuille exprimer "l'oiseau se pose sur la maison", le signe [oiseau] ayant une configuration en forme de bec, un emplacement sur la bouche. Le signe [maison] va tout d'abord être réalisé dans l'espace neutre [...] la configuration manuelle « main plate » va être conservée - comme " proforme » - et déplacée dans l'espace locatif [...]. Parallèlement [oiseau] va être signé, puis la configuration manuelle de [oiseau], avec le statut de proforme, va être incorporée au mouvement du verbe. Le point d'arrivée du verbe [se poser sur] sera alors la configuration «main plate» - proforme de [maison] - et réalisera, de façon iconique, la relation spatiale « sur ».

La notion de classificateur ou de proforme fait l'objet de nombreux débats et renvoie à des sens très différents notamment selon qu'elle est employée pour les structures de grande iconicité ou pour les signes standard (Millet, 2004 ; Voisin, 2008). Ce phénomène rappelle celui des classificateurs présents dans de nombreuses langues vocales du monde (Grinevald, 2002).

\subsection{Incorporation nominale}

Parmi les caractéristiques surprenantes de la LSF, figure la relation privilégiée entre le verbe et certains actants nominaux. Les verbes mentionnés dans la section 8.8 sont, à l'origine, formés de deux unités lexicales indépendantes. Ils se composent par exemple du lexème MANGER amalgamé respectivement aux lexèmes BAGUETTE, POMME et SPAGHETTI, pour BAGUETTE $\sim$ MANGER, POMME $\sim$ MANGER et SPAGHETTI MANGER ${ }^{42}$. 


$$
\begin{aligned}
& \text { subissent des modifications de la form } \\
& \text { verbes variables incluent : } \\
& \text { - les verbes directionnels réversibles } \\
& \text { - les verbes directionnels irréversibles } \\
& \text { - les verbes spatiaux } \\
& \text { - les verbes de localité }
\end{aligned}
$$


marquage des actants et pour indiquer la direction du procès ${ }^{46}$. L'existence de directionnels est également bien attestée dans les langues vocales. Certaines langues comme le jacaltec (langue maya), le japhug et le qiang (langues tibéto-birmanes) ont fortement grammaticalisé la direction au sein du prédicat verbal.

\section{8. « Haute résolution » sémantique des verbes}

64 La LSF a souvent recours à des spécifications sémantiques fines pour désigner certains verbes qui apparaissent en français comme des hyperonymes. Ainsi, on trouve des formes différentes pour le verbe manger:

- BAGUETTE MANGER (« manger avec des baguettes »)

- BANANE MANGER (« manger des bananes »)

- POMME MANGER (« manger des pommes »)

- SANDWICH MANGER (« manger un sandwich »)

- SPAGHETTI MANGER (« manger des spaghettis »)

- VIANDE MANGER (« manger de la viande »).

Il en va de même pour le verbe ouvrir qui est rendu par des signes différents selon les compléments associés au verbe en question :

- boUChE OUVRIR (« ouvrir la bouche »)

- cil OUVRIR (« ouvrir les yeux »)

- BOCAL COUVERCLE ÔTER (« ouvrir un bocal »)

- BOUTEILLE OUVRIR (« ouvrir une bouteille »)

- PAQUET $\sim$ DÉBALLER (« ouvrir un paquet »)

- FENÊTRE OUVRIR (« ouvrir la fenêtre »)

- FENÊTRE À GUILLOTINE OUVRIR (« ouvrir une fenêtre à guillotine »)

- PORTE OUVRIR (« ouvrir la porte »), etc. ${ }^{47}$

Dans les exemples ci-dessous, on constate pas moins de 8 verbes courants en LSF pour rendre le verbe ouvrir du français et la liste n'est pas exhaustive. Dans certains cas, il n'existe même pas d'hyperonyme. Ainsi, il n'y a en LSF aucun hyperonyme qui puisse correspondre au verbe ouvrir en français. S'il doit traduire ouvrir, un natif de LSF devra en l'absence de spécification, proposer plusieurs traductions selon le type d'objet.

La diversité et la richesse de formes que nous avons illustrées avec les traductions en LSF du verbe ouvrir n'est pas sans rappeler la diversité lexicale de certaines langues vocales comme c'est le cas dans des langues polynésiennes comme le futunien ou des langues kanak de Nouvelle Calédonie, comme le Xârâcùù ou des langues tibétobirmanes comme le tibétain. Voici quelques exemples :

- en futunien : kai « manger (en général) »; kina « manger des féculents »; fono'i « manger de la chair»; 'ota « manger cru»; taumafa « manger avec appétit»; pakalamu « manger avec bruit (gens) »; tapakā « manger avec bruit (cochons) », etc. ${ }^{48}$

- en tibétain: sha za «manger de la viande»; thug-pa 'thung "manger de la soupe (litt. boire)»; zho ldag « manger du yaourt (litt. lécher)»; rtsam-pa sgam « manger de la tsampa (sous forme de farine)»

- en xârâcùù : da «manger (en général) »; kê «manger des tubercules »; xwè " manger de la viande ou des condiments »; xwii « manger/mâcher de la canne à sucre ». 
Le type de spécificité sémantique que présentent certains verbes en LSF n'est donc pas un phénomène unique, mais il est typologiquement original et n'est pas attesté dans les langues de l'Europe.

\subsection{Absence de formes non-finies du verbe}

La LSF ne connait pas de sous-catégories morphologiques correspondant aux infinitifs, aux participes ou aux gérondifs. La nominalisation d'un verbe peut s'opérer par simple translation syntaxique, en déplaçant le verbe en position initiale qui est une position argumentale. Par exemple :

(12) LÀ-BAS + TRAVAILLER + FACILE + NON (« il n'est pas facile de travailler là-bas »)

(13) FUMER + SANTÉ + BON + NON (« fumer n'est pas bon pour la santé »)

Ce type de nominalisation est également attesté dans certaines langues vocales comme le chinois qui ne possède pas non plus d'infinitif, ni d'ailleurs de participe ou de gérondif.

\subsection{Absence de copule}

En général, les copules sont absentes en LSF. Les prédicats adjectivaux apparaissent également sans copule; par exemple :

(14) LUI (pointage) + PROFESSEUR (« il/elle [est] professeur »)

(15) LUI (pointage) + MALADE (« il/elle [est] malade »)

C'est aussi le cas dans de nombreuses langues (indonésien, russe ${ }^{49}$, etc.), contrairement au français, à l'anglais et à la plupart des langues européennes.

\subsection{Catégories adjectivales et spécificateurs de forme}

L'existence d'une classe d'adjectifs en LSF est admise par certains chercheurs ${ }^{50}$. Selon Risler (2007), on trouve notamment [des] lexèmes qui expriment des dimensions et des caractéristiques physiques graduables [...], tout comme c'est le cas dans de nombreuses langues vocales: grand versus petit, beau versus laid, gros versus maigre, long versus court. En LSF, ce type d'adjectif est appelé « spécificateur de taille et de forme " (STF). Les STF ont été décrits dans un certain nombre de travaux pour la LSF mais aussi pour l'ASL (Suppala, 1978, 1982 ; pour une synthèse de ces recherches, voir Risler, 2007).

Il est possible de donner des précisions sur la taille sans passer par un STF en modifiant simplement l'ampleur ou le mouvement du signe nominal qui constitue la tête du syntagme. On peut par exemple signer : LIVRE + ROUGE, en diminuant la taille du « livre » (zoom négatif) pour signifier «le petit livre rouge». Ce procédé augmentatif ou diminutif associé au substantif n'est pas sans rappeler les affixes éponymes dans les langues vocales; en espagnol, par exemple : librito «petit livre» et principito «petit prince ». En outre, certains adjectifs n'entrent pas dans la classe des STF, car ils ne 
spécifient pas une taille ou une forme particulière. C'est notamment le cas des adjectifs de couleur : BLANC, BLEU, NOIR, ROUGE, ROSE, etc.

D'un point de vue syntaxique, les diverses classes d'« adjectif » (les STF et les lexèmes adjectivaux) occupent en LSF une position postposée au nom. Toutefois, dans certaines LS, la position antéposée est aussi attestée.

\subsection{Classe d'adverbes}

Telle qu'elle est présentée dans les grammaires traditionnelles, mais aussi bien souvent dans des articles de linguistique contemporaine, la classe des adverbes constitue un ensemble totalement hétéroclite. En effet, elle rassemble des adverbes de manière (lentement, sérieusement, doucement), des quantifieurs (beaucoup, etc.), des intensifieurs (très, etc.), des marqueurs aspecto-temporels (déjà, souvent, etc.), etc. ${ }^{51}$. Les adverbes de manière constituent la catégorie prototypique de cette classe lexicale. D'un point de vue syntaxique, ils correspondent à des modifieurs verbaux et ont un fonctionnement différent des autres "adverbes", comme les intensifieurs et les quantifieurs.

La LSF confirme la nécessité de traiter ces unités de façon différente. Ainsi, les quantifieurs et les intensifieurs sont souvent réalisés en LSF par des opérateurs modaux (voir les catégories verbales ci-dessus) associés au mouvement (lent, rapide, ample, etc.) ou aux modalités indiquées par l'expression du visage. Par exemple :

(16) TRAVAILLER (l'action travailler est intense ; « elle travaille beaucoup »)

(17) TRAVAILLER (l'action travailler est soignée et la langue est serrée sur les dents ;

« elle travaille soigneusement »)

Le sens d'adverbes de manière tels que "lentement" ou «durement " peut être véhiculé par les mêmes procédés, notamment à l'aide des modalités liées à l'expression du visage ou bien par la rapidité d'exécution du signe :

(18) LIRE (l'action lire est lente ; « il lit lentement »)

79 Toutefois, dans le cas de certains adverbes de manière, il est aussi possible d'utiliser de vrais lexèmes adverbiaux : BIEN, FORT, RAPIDE, etc. Comme dans beaucoup de langues du monde, ce type d'adverbes est dérivé de leurs homologues adjectivaux, voire et dans certains cas, identiques : fort > fortement ou rapide $>$ rapidement, etc. ${ }^{52}$.

80 Concernant la catégorie lexicale des adverbes, celle-ci est limitée en LSF, car la fonction de modifieur verbal est souvent assurée par les modalités véhiculées par l'expression du visage.

\section{Conclusions}

81 Il s'agissait dans cet article de brosser les grands traits d'une typologie générale de la LSF. De nombreux points importants n'ont pas pu être traités, mais il en ressort d'ores et déjà un ensemble de conclusions. 

'analyse et la description de la LSF, mais aussi l'intérêt que présente cette dernière pour la typologie générale. Souhaitons que ce survol des catégories de la LSF contribue à attirer l'attention des linguistes et notamment des typologues sur les langues des signes trop souvent oubliées qui posent pourtant de véritables défis aux sciences du langage mais interrogent aussi la sociologie, l'ethnologie, l'anthropologie, la psychologie et l'épistémologie. cette langue dispose de certains types morphologiques et syntaxiques présents dans des langues vocales diverses et variées. La spatialisation et la directionnalité qui sont deux traits fondamentaux de la LSF ne sont pas sans correspondance dans certaines langues vocales même si en LSF, ces deux paramètres sont encore bien plus centraux dans le fonctionnement de la grammaire.

Nous avons brièvement abordé certaines caractéristiques typologiques générales de la LSF. Parmi elles, figurent, dans le domaine nominal, notamment l'absence de genre grammatical, l'absence d'article, l'optionalité du nombre ou encore l'existence de classificateurs ou proformes qui ne sont pas sans rappeler leurs homologues dans les langues vocales. Nous avons également vu qu'il y avait une grande richesse des pronoms qui sont réalisés par le biais des pointages et du regard.

Enfin, parmi les traits typologiques intéressants dans le domaine verbal, nous avons évoqué les phénomènes d'incorporation nominale, la spécificité des classes verbales (notamment les verbes réversibles), la «haute résolution » sémantique de certains verbes, l'absence de formes verbales non-finies ou encore l'absence de copule.

Nous avons montré qu'il est nécessaire de distinguer trois catégories lexicales en LSF : le nom, le verbe et, dans une moindre mesure, l'adjectif. Les autres catégories, celles des adverbes et des adpositions jouent un rôle marginal.

La liste des caractéristiques typologiques que nous avons évoquées n'est bien entendu, pas exhaustive. La LSF présente donc un grand intérêt pour la typologie des langues, car elle possède certaines caractéristiques très particulières à cause de son mode opératoire.

Loin des clichés encore très présents non seulement pour le grand public mais aussi pour un grand nombre de linguistes, la LSF possède une grammaire complexe qui n'est encore décrite que dans les grandes lignes.

\section{BIBLIOGRAPHIE}

Alegria, J. \& J. Leybaert (1986) Processus de lecture chez l'enfant sourd : une approche psycholinguistique, in Vivre sourd aujourd'hui... et demain, Bruxelles: Edirsa, p. 93-116.

Boutet, D. \& C. Cuxac (2008) Le signifiant gestuel : langues des signes et gestualité co-verbale, Cahiers de linguistique analogique, 5, p. 3-15.

TIPA. Travaux interdisciplinaires sur la parole et le langage, 34 | 2018 
Conrad, R. (1979) The Deaf schoolchild, language and cognitive function, London: Harper et Row, 370

p.

Cuxac, C. \& E. Antinoro-Pizzuto (2010) Émergence, normes et variation en langue des signes : vers une redéfinition conceptuelle, Langage \& société, 131, Sourds et langues des signes : norme et variations, p. 37-53.

Cuxac, C. (2001) Les langues des signes : une perspective sémiogénétique, AILE, 15, p. 3-9.

Cuxac, C. (2000) La langue des signes française : les voies de l'iconicité, Faits de Langues, 15-16, Paris-Gap: Ophrys, $391 \mathrm{p}$.

Cuxac, C. (1996) Fonctions et structures de l'iconicité des langues des signes : analyse descriptive d'un idiolecte parisien de la langue des signes française, Thèse de doctorat ès linguistique, Université Paris $\mathrm{V}$.

Cuxac, C. (1993) Iconicité des Langues des Signes, Faits de langues, 1, p.47-56.

DiFrancesca, S. (1972) Academic Achievement Test Results of a National Testing Program for Hearing Impaired Students, Washington, D.C.: Office of Demographic Studies, Gallaudet College.

Dryer, M. S. (2013a) Definite Articles, in Dryer, M. S. \& M. Haspelmath (eds.) The World Atlas of Language Structures Online (WALS Online). Leipzig: Max Planck Institute for Evolutionary Anthropology [en ligne]. Disponible sur : http://wals.info/chapter/37 (consulté le 11 août 2017).

Dryer, M. S. (2013b) Order of Subject, Object and Verb, in Dryer, M. S. \& M. Haspelmath (eds.) The World Atlas of Language Structures Online (WALS Online). Leipzig: Max Planck Institute for Evolutionary Anthropology [en ligne]. Disponible sur : http://wals.info/chapter/81 (consulté le 11 janvier 2018).

Ethnologue, Languages of the World (2013) Sign language [en ligne]. Disponible sur : https:// www.ethnologue.com/ et https://www.ethnologue.com/ethnoblog/ted-bergman/why-are-signlanguages-included-ethnologue (consulté le 24 août 2017).

Fabre, M. (2013) Analyse du fonctionnement cognitif d'adolescents sourds signeurs dans la pratique de l'écrit et via les Technologies de l'Information et de la Communication, Thèse de doctorat ès psychologie, Université d'Aix-Marseille.

Fradin, B. (2003) Nouvelles approches en morphologie, Paris: Presses Universitaires de France (Linguistique nouvelle), $347 \mathrm{p}$.

François, A. (2003) La sémantique du prédicat en Mwotlap (Vanuatu), Leuven, Paris: Peeters, 388 p.

Gaatone, D. (2008) Un ensemble hétéroclite : les adverbes de degré en français, in Durand, J., Habert, B. \& B. Laks (éds), Congrès Mondial de Linguistique Française, Congrès Mondial de Linguistique Française 2008 (CMLSF'08), Paris: Institut de Linguistique Française, 2495-2504 p. [en ligne]. Disponible sur : https://www.linguistiquefrancaise.org/articles/cmlf/pdf/2008/01/ cmlf08125.pdf (consulté le 25 août 2017).

Gallaudet University Library (2016) Sign languages of the world by name [en ligne]. Disponible sur : http://libguides.gallaudet.edu/content.php?pid=114804\&sid=991940 (consulté le 11 août 2017).

Garcia, B. (2017) Communication personnelle [courrier électronique].

Garcia, B. (2016) Scripturisation, grammatisation et modélisation linguistique à la lumière du cas des langues des signes, Les dossiers d'histoire, épistémologie et langage, 9, Écriture(s) et représentations du langage et des langues, p. 238-253. 
Garcia, B. \& M. Perini (2010) Normes en jeu et jeu des normes dans les deux langues en présence chez les sourds locuteurs de la Langue des Signes Française (LSF), Langage et société, 131, p. 75-93.

Girod, M., A. Vourc'h \& A.-C. Dufour (1990/1998) La langue des signes, dictionnaire bilingue élémentaire, volume 3, Vincennes: Éditions IVT, p. 218.

Goldin-Meadow, S. \& R. I. Mayberry (2001) How Do Profoundly Deaf Children Learn to Read? Learning Disabilities Research \& Practice, 16, p. 222-229.

Grinevald, C. (2002) Making sense of nominal classification systems: noun classifiers and the grammaticalization variable, in Wischer, I. \& G. Diewald (dir.) New reflections on Grammaticalization, Amsterdam, Philadelphia: John Benjamins Publishing Company, p. 259-275.

Hamm, M. (2012) Écrire sans entendre. Une exploration de la pratique de l'écriture chez quelques sujets sourds, devenus sourds et malentendants, Éducation et formation, 297, p. 138-153.

Hamm, M. (2010) L'apprentissage de l'écriture et de la lecture chez les personnes sourdes et malentendantes, Thèse de doctorat ès sciences du langage, Université de Strasbourg, 457 p.

Hamm, M. (2008) L'apprentissage de la lecture chez les enfants sourds : quels outils pédagogiques au service de quel apprentissage de la lecture ? Éducation \& Formation, 288, p. 37-44.

INSEE, « Handicaps-Incapacités-Dépendance, HID, 1998-1999 » (2007) Le handicap auditif en France. Apports de l'enquête Handicaps, incapacités, dépendance - 1998-1999, Sander, M.-S., Lelièvre, F., \& A. Tallec (dir.). Avec la collaboration de Dubin, J., Legent, F., Danet, S. \& J.-B.e Herbert, DREES, études et résultats, 589.

Jacquesson, F. (2008) Les personnes : morphosyntaxe et sémantique, Paris: CNRS, 370 p.

Kirtchuk, P. (2016) A united and integrative theory of language, Oxford: Peter Lang, 246 p.

Klima, E. S. \& U. Belligi (1979) The signs of language, Cambridge, MA, London: Harvard university press, $417 \mathrm{p}$.

LaPolla, R. J. (1993) Arguments Against 'Subject' and 'Direct Object' as Viable Concepts in Chinese, Bulletin of the Institute of History and Philology, 63, 4, p. 759-813.

Lavie, R.-J. (2007) Ordre des morphèmes et catégorisation nom-verbe dans une langue polysynthétique, l'inuktitut in séminaire Histoire et épistémologie des sciences du Langage, 18 juin 2007, Paris, Université Paris VII. Disponible sur : http://htl.linguist.univ-paris-diderot.fr/ slavie2.pdf (consulté le 25 août 2017).

Lejeune, F. (2004) Analyse sémantico-cognitive d'énoncés en Langue des signes française pour une génération automatique de séquences gestuelles, Thèse de doctorat ès informatique, Université de Paris XI, $260 \mathrm{p}$.

Lepot-Froment, C. \& N. Clerebaut (1996) L'enfant sourd. Communication et langage, Bruxelles: De Boeck Université, $672 \mathrm{p}$.

Meurant, L. (2008) Le regard en langue des signes. Anaphore en langues des signes de Belgique (LSFB) : morphologie, syntaxe, énonciation. Namur, Rennes: Presses Universitaires de Namur et Presses Universitaires de Rennes (Rivages linguistiques), $289 \mathrm{p}$.

Millet A. (2004) La langue des signes française (LSF) : une langue iconique et spatiale méconnue, Cahiers de l'APLIUT, 23, 2, p. 31-44.

Mithun, M. (1984) The evolution of noun incorporation, Language, 60, 4, p. 847-895.

Moyse-Faurie, C. (2015) Revitalisation des langues et Nouvelle Calédonie, Acte Sept, Colloque international de Biarritz, Chantier Sud-Nord, Théâtre du Versant, 20 novembre 2015. 
Mottez, B. (1977) À s'obstiner contre les déficiences, on augmente souvent le handicap. L'exemple des sourds, Sociologie et Société, 1, p. 20-32.

Mottez, B. (1976) La langue des signes aux États-Unis, Revue Générale de l'Enseignement des Déficients Auditifs, 4, p. 190-214.

Mugnier, S., Estève, I. \& A. Millet (2016) Dynamique du contexte sociolinguistique de la surdité en France : entre changement(s) et circularité, Glottopol, 27, p. 83-94.

Niederberger, N. (2004). Capacités langagières en langue des signes française et en français écrit chez l'enfant sourd bilingue : quelles relations? Thèse de doctorat ès psychologie, Université de Genève, $364 \mathrm{p}$.

Okrand, M. (1984) Star Trek III : The Search for Spock, Cinema Group Ventures, Paramount Pictures, États-Unis.

Padden, C. \& D. Clark (2005) Comment l'alphabet devint représenté en langue des signes, in Transler, C., Leybaert, J. \& J. E. Gombert, L'acquisition du langage par l'enfant sourd : les signes, l'oral et l'écrit, Marseille: Solal éditeur.

Plungian, V. A. (2000) Obšcaja morfologija : vvedenie v problematiku, [General morphology. Introduction to the problems], Moscow: Editorial URSS, $383 \mathrm{p}$.

Prinz, P., \& M. Strong (1998) ASL proficiency and English literacy within a bilingual deaf education model of instruction, Topics in Language Disorders, 18, 4, p. 47-60.

Risler, A. (2007) Les classes lexicales en LSF envisagées à partir de fonction adjectivale, Silexicales, 5, p. 103-125.

Risler, A. (2002). Point de vue cognitiviste sur les espaces créés en LSF : espace lexical, espace syntaxique. Lidil, 26, p. 45-61.

Sallandre, M.-A. (2003) Les unités du discours en Langue des Signes Française. Tentative de catégorisation dans le cadre d'une grammaire de l'iconicité, Thèse de doctorat ès Sciences du Langage, Université Paris 8, Saint-Denis, 2 volumes, 802 p.

Sallandre, M.-A. (2001) Va-et-vient de l'iconicité en Langue des Signes Française, A.I.L.E Acquisition et Interaction en Langue Étrangère, 15, p. 37-59.

Schön, J. (2008) La linguistique, Toulouse: Éditions Milan, 63 p.

Stokoe W. (1960) Sign Language Structure : An Outline of the Visual Communication System of the American Deaf, University of Buffalo, Studies in Linguistics, Occasional Paper, 8, 78 p.

Tersis, N. (2008) Forme et Sens des Mots du Tunumiisut : lexique inuit du Groenland oriental. Traduit de l'anglais par R. Boyd, Louvain, Paris: Peeters, 590 p.

Timmermans, N. (2005) Le statut des langues des signes en Europe, Conseil de l'Europe, 172 p.

TIPA (2017). Convention de transcription des énoncés signés en français écrit [en ligne]. Disponbile sur : https://tipa.revues.org/1723 (consulté le 13 septembre 2017).

Tournadre, N. (2014b/2016) Le Prisme des Langues, essai sur la diversité linguistique et les difficultés des langues, Paris: L'Asiathèque, $349 \mathrm{p}$.

Tournadre, N. (2014a) Arguments against 'Subject' and 'Direct Object' as viable concepts in Tibetan: Revisiting the syntactic categories for Classical Tibetan as well as the modern Tibetic languages, paper presented at the 20th Himalayan Languages Symposium, NTU, Singapore, 16-18 July 2014. 
Tournadre, N. \& R. J. LaPolla (2014) Towards a new approach to evidentiality, Linguistics of the Tibeto-Burman Area [LTBA], 37, 2, p. 240-263.

Tournadre, N. (2010) The Classical Tibetan cases and their transcategoriality From sacred grammar to modern linguistics, Himalayan Linguistics Journal, 9, 2 p. 87-125.

Transler, C, (2005) Comment l'enfant sourd développe puis utilise le principe du B-A/ba/, in Transler, C, J. Leyaert \& J. E. Gombert (éds), L'acquisition du langage par l'enfant sourd : les signes, l'oral et l'écrit, Marseille: Solal, p. 317-326.

Voisin, E. (2008) Analyse syntaxique et formalisation d'énoncés en langue des signes française, Thèse de doctorat ès sciences du langage, Université Michel de Montaigne de Bordeaux III, 213 p.

Walter, H. \& A. Lorca (2001) Question à... Henriette Walter, L'Express [en ligne]. Disponible sur : http://www.lexpress.fr/culture/livre/honni-soit-qui-mal-y-pense_804257.html (consulté le 28 juillet 2017).

Wikipedia (2015) Liste des langues des signes [en ligne]. Disponible sur : https://fr.wikipedia.org/ wiki/Liste_des_langues_des_signes (consulté le 24 août 2017).

Wittmann, H (1991) Classification linguistique des langues signées non vocalement, Revue québécoise de linguistique théorique et appliquée, 10, 1, p. 215-288.

Wrightstone, W. J., Aronow, M. S. \& S. Moskowitz (1963) Developing reading test norms for deaf children, American Annals of the Deaf, 108, p. 311-316.

Yau, S. (2004) Les sourds chinois et l'écriture idéographique, in Le bilinguisme : bien lire, aimer lire. Les différentes pratiques. Actes de la journée d'étude du 21 novembre 2004, GERS, Paris, p. 67-78.

Yau, S. (1993) Iconicité à deux niveaux : de la forme à la motivation, Faits de langues, 1, p. 57-67.

Yau, S. (1992) Création gestuelle et débuts du langage : création de langues gestuelles chez des sourds isolés, Paris: Éditions Langages croisés, 501 p.

Zamenhof, L. L. (1887/1897) Langue internationale « Esperanto » : manuel complet avec double dictionnaire, traduit du russe par L. de Beaufront, Paris: H. le Soudier, 168 p.

Zeshan, U. (2006) Interrogative and Negative Constructions in Sign Languages, Nijmegen: Ishara press, $365 \mathrm{p}$.

\section{NOTES}

1. Certains pays, comme le Royaume-Uni, n'ont pas de constitutions écrites ; ils ne peuvent donc pas reconnaître leur(s) langue(s) des signes sur le plan constitutionnel (voir notamment Timmermans, 2005).

2. «Elle [la "langue" des signes] a pour objectif de compenser la langue véritable/ordinaire. Elle est d'une certaine façon proche de la "langue" des signaux maritimes utilisée pour la communication navale lorsque les distances étaient trop grandes pour communiquer autrement" (notre traduction). Ces affirmations de Kirtchuk (2016) nous semblent aller à l'encontre même de sa théorie «biologique » du langage à laquelle nous adhérons. Notons au passage que cette vision n'est pas très éloignée de celle qui prévalait jusque dans les années soixante-dix du $20^{\text {ème }}$ siècle, comme le rappelle Wittmann (1991) en critiquant cette position dans son article de référence.

3. Elles diffèrent en cela de l'esperanto ou du klingon qui sont des langues artificielles, inventées respectivement par Zamenhof (1887) et Okrand (1984). 
4. Le terme de «chérologie » a été proposé par Stokoe (1960) pour étudier les unités minimales non significatives nommées «chérèmes" qui correspondent aux phonèmes dans les langues vocales. Ces termes ne sont presque plus utilisés depuis Stokoe (1960); voir la section 4.1 intitulée « Le paramétrage ».

5. Par conséquent, nous sommes contraints de limiter notre analyse à cette langue, sans présumer de sa validité pour d'autres langues des signes.

6. Chiffre établi par Ethnologue, Languages of the World (2013). Si on inclut les LS émergentes, interindividuelles ou micro-communautaires, leur nombre est nettement plus élevé. Ainsi, Wikipedia (2015) fournit une liste de 185 langues des signes, et selon Gallaudet University Library (2016), il y aurait « 271 identified sign languages, dialects, and other sign systems». Tous ces chiffres doivent être pris avec précaution, tant que l'on n'a pas de description précise des langues en question.

7. Dans le cas bien sûr où les signeurs ont recours aux signes standard de leur langue respective. Ils ont toujours l'option pour une communication de base de passer par la grande iconicité. Ainsi, les signes standard de la langue des signes tibétaine (LST), notamment, sont incompréhensibles à des signeurs de LSF. Si une compréhension de base s'instaure, elle ne se fonde pas sur les signes standard de la LSF ou de la LST, mais sur la recherche d'un consensus à partir de la grande iconicité. De la même façon qu'il n'y a pas d'intercompréhension permettant une conversation orale aisée entre certaines formes d'arabe parlé ou darija (arabe du Mashreq, arabe du Maghreb et arabe des Bédouins), il est cependant évident que la conversation devient tout à fait possible si les différents locuteurs ont une connaissance raisonnable de la fusha (langue écrite).

8. En LSF, le terme CAFÉ est réalisé par un signe qui rappelle un moulin à café moulinant. De ce point de vue, les langues des signes ressemblent tout à fait aux langues vocales qui sont dans une certaine mesure des musées de concept. Aussi, le terme chu-tshod en tibétain désignant par une montre ou une horloge signifie " mesure à eau », ce qui fait allusion aux antiques clepsydres. De même, le terme chauffeur en français est une référence au fait qu'il fallait préalablement chauffer la voiture, avant de la démarrer.

9. Ainsi, il semble que l'on trouve des différences dans les «lignes du temps » qui servent à représenter le temps grammatical dans diverses LS.

10. Cela consiste à adopter des stratégies iconiques générales et non une langue des signes particulière, comme nous l'avons indiqué à la note 7 .

11. C'est nous qui soulignons.

12. De ce point de vue, les études de cette auteure, notamment sur les constructions interrogatives et négatives, font figure de travaux pionniers.

13. Bien que la LSSF utilise des signes standard de la LSF, sa syntaxe est plus semblable à celle du français qu'à la LSF. Cette langue hybride est souvent pratiquée par des non-natifs qui ne maîtrisent pas la syntaxe de la LSF.

14. Communication personnelle de B. Garcia (avril 2017). Cette situation n'est pas sans rappeler celle des créoles avec le continuum entre les basilectes et les acrolectes.

15. Il n'existe aucun système standard de transcription écrite des langues signées (voir Garcia 2016). Cela tient à la complexité tridimensionnelle et à la multimodalité du système. Faute de transcription écrite standard, les corpus en LSF sont généralement présentés à partir d'enregistrements vidéo. Toutefois, certains systèmes de transcription ont été proposés comme l'ISWA ; ils sont en cours de développement (voir par exemple Garcia \& Sallandre, 2013).

16. Voir notamment INSEE «Handicaps-Incapacités-Dépendance, HID, 1998-1999»; Niederberger, 2004 ; Fabre, 2013. Voir aussi les travaux d'Alegria \& Leybaert, 1986; LepotFroment \& Clerebaut, 1998 ; Transler, 2005 en Belgique ; de Conrad, 1979 en Grande-Bretagne ; de Wrighstone, Aronow et al., 1963 ; DiFrancesca, 1972; Prinz \& Strong, 1998; Goldin-Meadow \& Mayberry, 2001 aux États-Unis.

TIPA. Travaux interdisciplinaires sur la parole et le langage, 34 | 2018 
17. Rappelons qu'en 2014, l'écrasante majorité des langues du monde ne sont pas dotées d'écriture (Tournadre, 2014b/2016).

18. Notamment des discours faisant une grande utilisation de l'alphabet dactylologique.

19. Il ne faut bien entendu pas confondre la question de l'iconicité présente dans les unités lexicales et celle qui fonde les structures de transfert. Dans ces dernières, l'iconicité est fondamentale.

20. Dans certains cas, les mouvements labiaux (mouthing en anglais) désambigüisent des signes homophones. En langue des signes italienne, par exemple, il y a un seul signe HERBE pour différentes herbes; chaque herbe se différencie en lecture labiale: oregano (origan), basil (basilic), etc. (Padden \& Clark, 2005 : 76).

21. Le terme de morphème pour décrire les langues vocales est abandonné dans certaines théories de la morphologie (voir Fradin, 2003).

22. Communication personnelle de B. Garcia (avril 2017).

23. Si dans le cas des langues vocales, le fait de hausser la voix pour exprimer la colère est universel, en LSF, c'est surtout la distance réduite entre les signeurs et la vitesse, l'amplitude et l'intensité d'exécution des signes associés à une expression faciale menaçante qui signalent la dispute. En outre, des cris ou des sons peuvent être émis ; le sourd n'est donc pas muet comme le suggère l'expression « sourd-muet ».

24. En visée illustrative, chacune des deux mains assume souvent des rôles sémantiques différents (Cuxac, 2001).

25. Le premier est partiellement iconique et les deux autres sont phonétiques, mais le syllabaire note des syllabes tandis que l'alphabet note des consonnes ou des voyelles.

26. Voir Tournadre, 2014b/2016: 283.

27. Voir par exemple Dryer (2013b).

28. Voir aussi Yau, 1992 : 85-86.

29. Là encore, l'influence de l'animacité et d'autres paramètres sémantiques sur la syntaxe est attestée dans de nombreuses langues vocales.

30. Nous utilisons les petites majuscules pour dénoter les unités lexicales (signes standard). Contrairement à de nombreux chercheurs travaillant sur la LS, nous n'avons pas ici travaillé sur un corpus avec des arrêts sur images et avons préféré utiliser un système de gloses proche de celui utilisé par Klima et Bellugi (1979) ou Zeshan (2006). De tels systèmes de glose proches de celles utilisées dans les LV ont été critiqués (voir notamment Garcia, 2016). La langue d'annotation qui sert à la fois à représenter les signes et les annoter n'est pas sans induire des effets spécifiques et indésirables. Ces effets ne sont pas absents dans les LV; les notations en API ainsi que les gloses peuvent être influencées par la langue maternelle du linguiste et bien entendu par les modèles théoriques utilisés.

31. Nous nous référons à la convention de transcription des énoncés signés en français écrit de TIPA (2017).

32. La supplétion consiste à utiliser des formes lexicales différentes à l'intérieur d'un même paradigme. On peut mentionner le cas classique du verbe aller en français qui utilise trois verbes latins différents pour former les divers temps : j'irai (ire), j'allais (ambulare), je vais (vadere). La conversion consiste à utiliser une forme associée à une catégorie lexicale pour désigner une autre catégorie. Par exemple, le verbe drink en anglais peut être utilisé comme un substantif. Inversement le nom bridge peut être utilisé comme un verbe sans que la forme ne soit modifiée. La supplétion consiste à utiliser des formes lexicales différentes à l'intérieur d'un même paradigme. On peut mentionner le cas classique du verbe aller en français qui utilise trois verbes latins différents pour former les divers temps : j'irai (ire), j'allais (ambulare), je vais (vadere). La conversion consiste à utiliser une forme associée à une catégorie lexicale pour désigner une autre catégorie. Par exemple, le verbe drink en anglais peut être utilisé comme un substantif.

TIPA. Travaux interdisciplinaires sur la parole et le langage, 34 | 2018 
Inversement le nom bridge peut être utilisé comme un verbe sans que la forme ne soit modifiée. Ces deux fonctions sémantiques sont également présentes dans les langues vocales.

33. Même dans les langues vocales, la distinction entre affixes (préfixes, suffixes, circonfixes, infixes) et clitiques (préposés, postposés, interclitiques, etc.) n'est pas toujours aisée. De nombreux segments qui étaient traditionnellement considérés comme des affixes ou à l'inverse des mots autonomes (adpositions, articles, etc.) sont maintenant décrits comme étant des clitiques (Plungian, 2000). C'est le cas, par exemple, des articles du français ou encore des prépositions du russe ou bien des suffixes casuels du tibétain (Tournadre, 2010).

34. C'est-à-dire le marquage des arguments au niveau verbal.

35. Même ce point fait l'objet de débat : Garcia (communication personnelle, avril 2017) déclare : En LSF, la majorité des unités lexicales ne sont pas identifiables comme nominales ou verbales en dehors du discours.

36. Par exemple, l'utilisation de possessifs en LSF - MON, TON, SON, etc. - induit une interprétation nominale, tandis que des marques de temps-aspect ou de mode comme FINI induisent généralement une lecture prédicative verbale. Ainsi, LIVRE + MON (« mon livre ») et LIRE + FINI ( j'ai lu »); mais LIVRE + FINI ( " j'ai fini mon livre ») et LIRE + MON (" ma lecture »).

37. Certains chercheurs signalent que la position antéposée du pointage est également attestée.

38. Il est intéressant de noter que cette absence de marque pour la première personne s'étend aux questions adressées au co-signeur par anticipation sur la réponse. Ce même phénomène d'anticipation est attesté dans certaines langues vocales comme les langues tibétiques (Tournadre et LaPolla, 2014).

39. Mais, généralement, après NOUS CINQ ou vous CINQ, c'est deux tridents qui se ferment devant soi.

40. L'inclusif implique que l'interlocuteur est inclus dans les personnes désignées. Ainsi, la forme inclusive "nous trois » inclut le locuteur, l'interlocuteur et une troisième personne, tandis que l'exclusif «nous trois» désigne trois personnes dont le locuteur et deux autres personnes excluant l'interlocuteur. En LSF, cette opposition est réalisée par le pointage. De nombreuses LV ont aussi des marques spécifiques pour noter l'inclusif et l'exclusif. C'est le cas notamment de nombreuses langues tibétiques, dravidiennes ou austronésiennes.

41. Il arrive aussi que les démonstratifs donnent lieu à l'apparition de pronoms relatifs comme en anglais « that ».

42. Nous ajoutons le tilde $\sim$ afin d'indiquer cette relation morphologique complexe.

43. Il est préférable pour la LSF et les LS en général d'utiliser des rôles grammaticaux dérivés de rôles sémantiques comme ceux d'agent $(\mathrm{A})$, de patient $(\mathrm{P})$ ou d'actant unique (U). Ces étiquettes sont plus pertinentes que les étiquettes purement syntaxiques de Sujet et d'Objet (voir Yau, 1992).

44. L'incorporation d'instrument et de lieu est attestée dans certaines langues amérindiennes (Mithun, 1984 ; voir Voisin, 2008).

45. Cette classification est adaptée à partir de celle proposée par Voisin (2009).

46. C'est-à-dire le sémantisme du verbe.

47. Cuxac (2000) a décrit ce phénomène comme des «stratifications sémantiques fines ».

48. Cf. Moyse-Faurie (2015).

49. La copule est absente uniquement au présent.

50. Notons au passage que la catégorie d'adjectif n'est pas considérée comme une classe lexicale universelle. Pour certaines langues comme le chinois, elle est inexistante et pour d'autres, au contraire, elle est hypertrophiée, comme c'est le cas dans la langue russe qui possède plusieurs classes d'adjectifs et en fait un très grand usage.

51. Même les sous-classes d'adverbes sont hétérogènes (voir notamment, Gaatone, 2008). 
52. C'est le cas par exemple en latin ou en russe. Les adverbes sont parfois identiques aux adjectifs comme en tibétain ou correspondent à un redoublement de la forme adjectivale comme en persan.

\section{RÉSUMÉS}

Qu'entend-on par «langues des signes »? Quelles sont leurs spécificités ? Quel est l'intérêt des langues signées pour la linguistique ? Alors que les sciences du langage se sont considérablement développées durant ces dernières décennies, permettant de mieux comprendre le fonctionnement des langues et leurs variations, les langues des signes posent encore beaucoup de questions notamment pour la typologie des langues. L'objectif de cet article est de montrer que la langue des signes française (LSF), au-delà de ses spécificités structurelles et fonctionnelles, partage des caractéristiques typologiques que l'on retrouve dans certaines familles de langues vocales ainsi que dans des langues écrites de type logographiques.

What do we mean by 'sign languages'? What are the specificities of sign languages? In what way; might sign languages contribute to general linguistics? Linguistics has undergone remarkable developments during the last decades, however Sign languages constitute a challenge particularly for the field of linguistic typology. The aim of this paper is to show that, beyond its structural and functional specificities, French Sign Language (langue des signes française, LSF), shares many typological characteristics found in many spoken languages of the World as well as in some written logographic systems.

General typology sheds new light on Sign languages and shows that beyond functional specificities related to the visual-gestural modalities, this language displays a number of morphological and syntactic characteristics attributed to many spoken languages. There is allegedly at least 136 Sign languages in the World but some sources provide a much higher figure of nearly three hundred. The genetic classifications of Sign languages are still imprecise and the grouping of SL into families are not widely accepted. A great number of SLs are not mutually intelligible. SLs are minority languages without precise geographic location. They are silent languages that share some features of the written logographic systems but do not have any written system. SL makes use of two entirely different types of "signs": standard signs and transfers. Signs may be analyzed according to several parameters. Each area of the signing space is significant. Sign languages are spatial and «situational ». The preferred syntactic order in LSF is: Agent-Patient Predicate ( SOV »). Concerning lexical categories, it is necessary to distinguish three categories in LSF: nouns, verbs and to a lesser extent, adjectives. Other categories such as adverbs or adpositions play a marginal role. LSF does not have any grammatical gender or article. Number is optional. There are various types of pronouns which are essentially marked through pointing signs and eye-gaze. Among the typological features that we discuss in this article, we can recognize classifiers or pro-forms, nominal incorporation, various verb classes, the high semantic resolution of some verbs, the absence of non-finite forms of verbs, the absence of copula as well as the adjectival category. The list of typological characteristics presented here is of course not exhaustive. Moreover, in order to allow a typological comparison with spoken languages, we focused on "Standard signs " and did not discuss in depth iconicity, which is a fundamental feature of LSF and other sign languages. In sharp contrast with the clichés and stereotypes shared by the general public as well as by a number of linguists, LSF has a complex 
grammar which has not been described in detail. This paper strives to show the necessity of a typological approach for the analysis and description of LSF, but it also demonstrates the great interest of this language for general typology. Let's hope that this overview of categories in LSF will contribute to draw attention to sign languages from a growing number of linguists and particularly typologists. These languages are often forgotten in the general discussion about Human language despite the fact that they offer a great challenge for linguistic theories as well as other fields of Human sciences such as sociology, anthropology, psychology and epistemology.

\section{INDEX}

Mots-clés : langues des signes, LSF, caractéristiques typologiques, catégories lexicales, catégories grammaticales, linguistique générale

Keywords : Sign languages, French Sign Language (LSF), typology, word classes, grammatical categories, general linguistics

\section{AUTEURS}

\section{NICOLAS TOURNADRE}

Aix-Marseille Université, Institut Universitaire de France, CNRS, LACITO, Villejuif, France Nicolas.tournadre@univ-amu.fr

\section{MÉLANIE HAMM}

Aix-Marseille Université, CNRS, LPL, Aix-en-Provence, France

Melanie.hamm@univ-amu.fr 